\title{
Preprocessing and Feature Extraction for Psoriasis Images Based on Discrete Wavelet Transform
}

\author{
Raniah Ali Mustafa ${ }^{1}$ Haitham Salman Chyad $^{2}$ \& Rafid Aedan Haleot $^{3}$ \\ ${ }^{1-3}$ Mustansiriyah University,
} College of Education, Department of Computer Science

Iraq

\begin{abstract}
This paper, proposes the pre-processing methods use a small neighborhood of a pixel in the input image to get a new brightness value in the output image. Such pre-processing operations are also called filtration. in this paper, we start with the image using a medical case for psoriasis image after change it to gray state implemented under the transform domain (i.e frequency), using wavelet transform then use three filters sharpening, Sobel, and Laplace filter. after make proposed by computing PSNR for each state to show the effect of it. Then extract features through an apply a set of measures (Energy, Entropy, Standard deviation, Variance, Mean) of low low sub-image. The proposed system was implemented on the medical case for psoriasis image dataset, some of them were obtained from the hospitals and the other was obtained from the dataset (Light Field Image of Dataset skin Lesions), available on the Internet and the proposed system implemented in programing language Visual Basic 6.0.
\end{abstract}

Key Words: Psoriasis Images, Wavelet Transform, Haar Basis Filter, Sharpening, Soble, Laplacian.

\section{INTRODUCTION}

Wavelet transforms have become one of the most important and powerful tool in image processing, data compression, and signal processing. and any operation that is aimed at modifying the visual impression of image? traditional image sharpening methods usually introduce over shooting along the edges, and do not perform well for sharpening an image resulting from high magnification zooming. the fundamental idea of image sharpening is to add to the input signal a high-pass filtered version of the signal itself. Than a the sobel operator performs a 2-D spatial gradient measurement on an image and so emphasizes regions of high spatial frequency that correspond to edges. typically, it is used to find the opproximate absolute gradient magnitude at each point in an input gray scale image. finally, the laplacian is a 2-D isotropic measure of the 2nd spatial derivative of in image. the laplacian of an image highlights regions of rapid intensity change and is therefore often used for edge detection (see zero crossing edge detectors). the laplacian is often applied to an image that has first been smoothed with something approximating a Gaussian smoothing filter in order to reduce its sensitivity to noice, and hence the two variants will be described together here. the operator normally takes a single gray level image as input and produces another gray level image as output. In this paper an algorithm is invested to preprocessing image to detect or recognize psoriasis place using three types of filters. the rest of this paper start with an overview about psoriasis, DWT section accommodate with brief definition basic filters used section three state related works with our paper [1].

\section{PSORIASIS IMAGES}

Psoriasis is a chronic skin disease characterized by red patches on the skin, often accompanied by silvery-white scales of dead skin cells. It is not contagious. Psoriasis is believed to be an autoimmune disease, meaning the body's defense system attacks healthy cells in the body. This creates skin cells that mature and die in less than a week, which is extremely rapid considering normal skill cells mature and replace dead ones usually in a month's time [2]. 


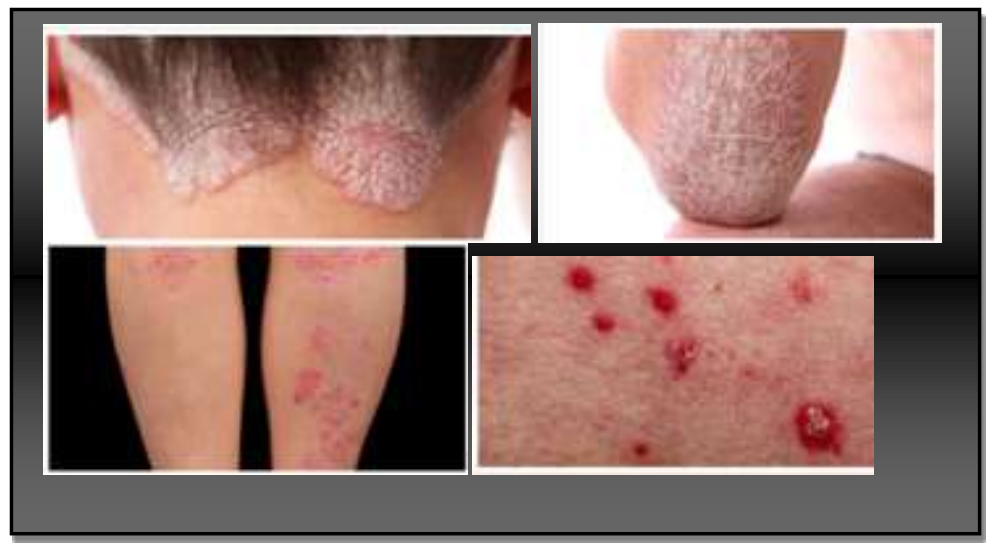

Figure 1.1: stages of psoriasis

\section{WAVELET TRANSFORM}

A "wave" is generally defined as an oscillatory function from space or time, such a sinusoid. "Wavelet" phrase of a "small wave", that contains energy intensified in time to give a tool for the analyses of non-stationary, transient, or time-varying phenomena [3]. A wavelet is a mathematical function utilized to split continuous-time signal or a given function into various scale components. Figure (1.2) shows the wave (sinusoid) and the wavelet [4].

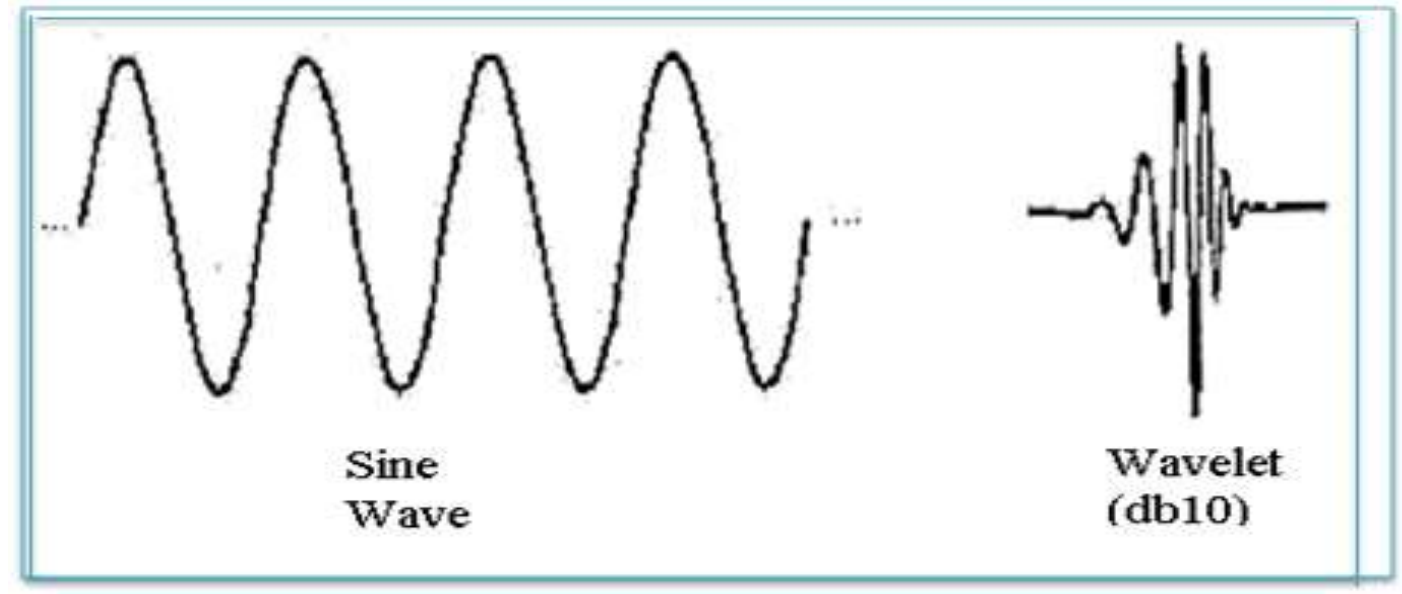

Figure 1.2: A Wave and Wavelet [4]

Wavelet transform (WT) provides powerful signal analysis tools, which will be generally utilized within image compression, de-noising, feature extraction, detection, recognition, and image retrieval applications. Wavelet decomposition is the most widely utilized multi-resolution technicality in image processing. Due to the excellent time-frequency localization discriminative. WT provide a powerful mathematical tool. Images have typically locally differing statistics that result of various combinations of unexpected features such edges, of relatively low-contrast homogeneous regions and of textured regions. However, like spatial and variability non-stationary defiance each single statistical description, the multi-resolution components is more easily handled. WT could be completed for each translation and scale [5].

There are two kinds of WT; continuous wavelets transform (CWT) and discrete wavelet transforming (DWT). The main difference among CWT and DWT is that CWT uses each probable scale and translation and DWT use an explicit subset of scale and translation values [3]. 


\subsection{HAAR BASIS FILTER}

The Haar basis filter consists of high pass filter (HPF) and Low pass filter (LPF) are defined in equations following:

$$
\begin{aligned}
& \text { HPF: } 1 / \sqrt{2}\left[\begin{array}{ll}
1 & -1
\end{array}\right], \\
& \text { LPF: } 1 / \sqrt{2}\left[\begin{array}{ll}
1 & 1
\end{array}\right],
\end{aligned}
$$

The HP and LP filters are so-called the decomposition filters for they separate the picture down or decompose the picture to approximation and detailed, coefficients respectively. The convolution with the band pass filter in a certain direction results in socalled details picture and the convolution with the LPF results in a so-called approximation picture [6].

LL band (approximation band) is the result of applying LPF in horizontal and vertical directions and its filter derived as follows:

$$
\mathrm{LL}=\frac{1}{\sqrt{2}}\left(\begin{array}{ll}
1 & 1
\end{array}\right)^{\mathrm{t}} \cdot \frac{1}{\sqrt{2}}\left(\begin{array}{ll}
1 & 1
\end{array}\right)=\frac{1}{2}\left(\begin{array}{ll}
1 & 1 \\
1 & 1
\end{array}\right)
$$

LH band (detail band) is the result of applying horizontal LPF and vertical HPF and its filter is derived as follows:

$$
\mathrm{LH}=\frac{1}{\sqrt{2}}\left(\begin{array}{ll}
1 & 1
\end{array}\right)^{\mathrm{t}} \cdot \frac{1}{\sqrt{2}}\left(\begin{array}{ll}
1 & -1
\end{array}\right)=\frac{1}{2}\left(\begin{array}{cc}
1 & -1 \\
1 & -1
\end{array}\right),
$$

HL band (detail band) is the result of applying horizontal HPF and vertical LPF and its filter is derived as follows:

$$
\mathrm{HL}=\frac{1}{\sqrt{2}}\left(\begin{array}{ll}
1 & -1
\end{array}\right)^{\mathrm{t}} \cdot \frac{1}{\sqrt{2}}\left(\begin{array}{ll}
1 & 1
\end{array}\right)=\frac{1}{2}\left(\begin{array}{cr}
1 & 1 \\
-1 & -1
\end{array}\right)
$$

$\mathrm{HH}$ band (detail band) is the result of applying horizontal and vertical HPF and its filter is derived as follows:

$$
\mathrm{HH}=\frac{1}{\sqrt{2}}\left(\begin{array}{ll}
1 & -1
\end{array}\right)^{\mathrm{t}} \cdot \frac{1}{\sqrt{2}}\left(\begin{array}{ll}
1 & -1
\end{array}\right)=\frac{1}{2}\left(\begin{array}{lr}
1 & -1 \\
-1 & 1
\end{array}\right)
$$

The transformed bands (LL scaling bands and three wavelet bands HL, LH, and HH) can be obtained by applying above filters to each $(2 \times 2)$ adjacent pixel of the whole picture pixel. This approach is called Fast Mallat transform algorithm [6].

\begin{tabular}{|c|c|c|}
\hline LL2 & HL2 & HL1 \\
\hline LH2 & HH2 & \\
\hline & HH1 \\
\hline
\end{tabular}

Figure 1.3: Two level Wavelet Sub Band Decomposition

\subsection{DISCRETE WAVELET TRANSFORM DECOMPOSITION}

The DWT for 2-dimensional images $x[m, n]$ can be identically defined through applying 1-dimensional DWT to every dimension $\mathrm{m}$ and $\mathrm{n}$ independently: $\mathrm{DWT}_{\mathrm{n}}\left[\mathrm{DWT}_{\mathrm{m}}[\mathrm{x}[\mathrm{m}, \mathrm{n}]]\right]$. 2-dimensional WT decomposes an image to "sub bands" which are centralized in orientation and frequency. WT is formed through passing the image by sequences of filter bank phases. One phase is demonstrationed in Figure (1.4). In which an image is initially filtered in the horizontal direction. 


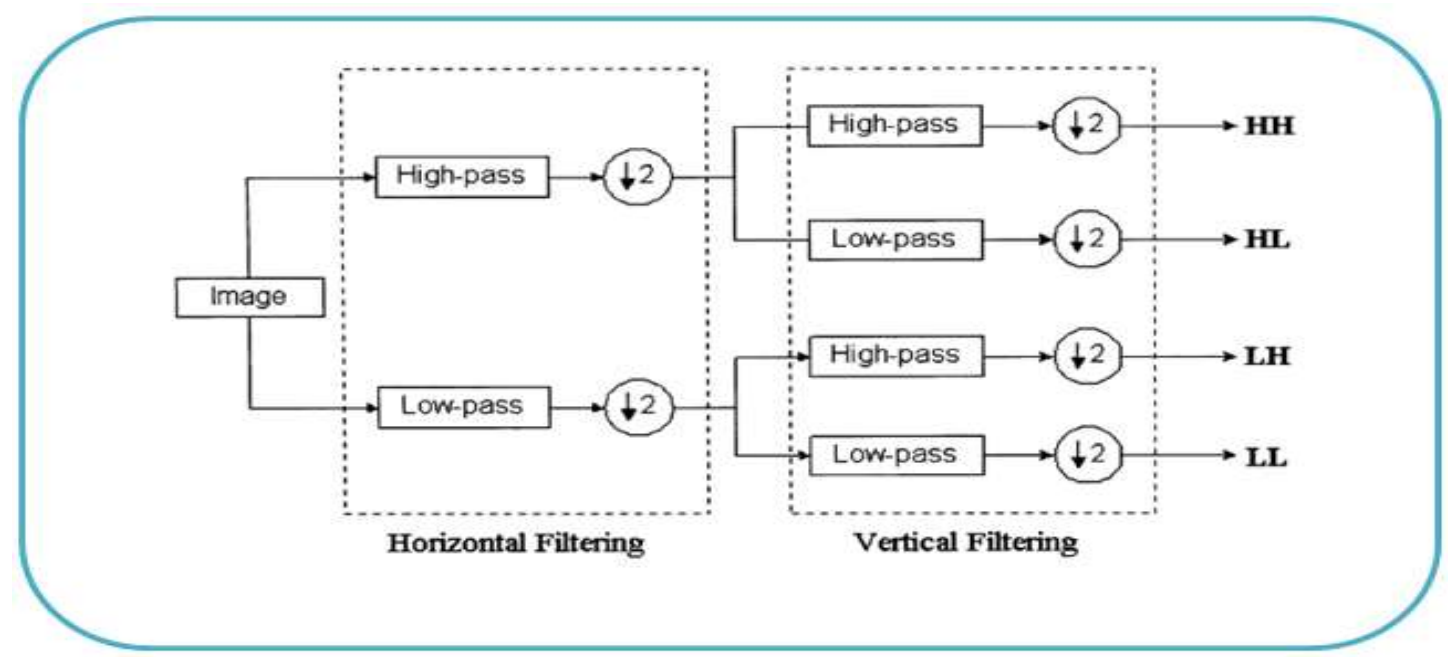

Figure 1.4: Illustration of 2-D Wavelet Transforms

The scaling function (LPF) \& wavelet function (HPF) are limited pulsation reply filters. In other words, the output at every point relies just on a limited part of the input. The filtered outputs are then down sampled by a factor of $2(\downarrow 2)$ in the horizontal direction. Those signals are then each filtered through a similar filter pair in the vertical direction. Eventually decomposite the image to 4 sub bands indicated by (LL, HL, LH, HH). Each of those sub bands could be thought of as a smaller version of the image which represents various image properties. The Low-Low is a coarser approximation to the original image. Low High \& High Low record the differences of the image along vertical and horizontal directions, consective. High High offers the high frequency component of the image. 2-Level decomposition could then be conducted on the Low Low sub band. Figure (1.5) demonstrates two-Level wavelet decomposition [7,8].

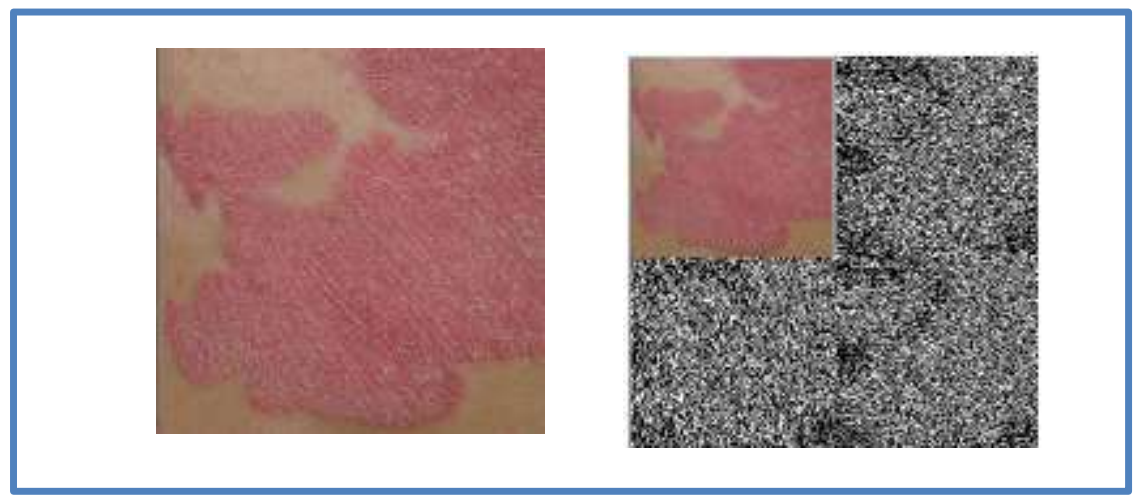

Figure 1.5 : (a) Original Image (b) Two-Level Wavelet Decomposition

\section{SHARPENING FILTER}

Most image sharpening software tools work by applying something called an "unsharp mask," which despite its name, actually acts to sharpen an image. In a nutshell it works by exaggerating the brightness difference along edges within an image Note that while the sharpening process isn't able to reconstruct the ideal image above, it is able to create the appearance of a more pronounced edge. The key to effective sharpening is walking the delicate balance between making edges appear sufficiently pronounced, while also minimizing visible under and overshoots (called "sharpening halos"). Sharpening is a key post-production step when finishing your images. An Edge mask is a simple layer that accentuates just the edges in your photo to selectively sharpen it see figure (1.6) 


\section{Figure 1.6: sharpening filter Output}

The Find Edges filter generates a fast and accurate mask that's used to apply sharpening where an image needs it most; on the edges [9].

\section{SOBEL FILTER}

In theory at least, the operator consists of a pair of $3 \times 3$ convolution kernels as shown in Figure (1.7). One kernel is simply the other rotated by $90^{\circ}$. This is very similar to the Roberts Cross operator.

\begin{tabular}{|l|l|l|}
\hline-1 & 0 & +1 \\
\hline-2 & 0 & +2 \\
\hline-1 & 0 & +1 \\
\hline
\end{tabular}

$\mathrm{Gx}$

\begin{tabular}{|c|c|c|}
\hline+1 & +2 & +1 \\
\hline 0 & 0 & 0 \\
\hline-1 & -2 & -1 \\
\hline
\end{tabular}

Gy

Figure 1.7: Sobel convolution kernels

These kernels are designed to respond maximally to edges running vertically and horizontally relative to the pixel grid, one kernel for each of the two perpendicular orientations. The kernels can be applied separately to the input image, to produce separate measurements of the gradient component in each orientation (call these $G x$ and $G y$ ). These can then be combined together to find the absolute magnitude of the gradient at each point and the orientation of that gradient. The gradient magnitude is given by:

$$
|G|=\sqrt{G x^{2}+G y^{2}}
$$

Typically, an approximate magnitude is computed using:

$$
|G|=|G x|+|G y|
$$

which is much faster to compute.

The angle of orientation of the edge (relative to the pixel grid) giving rise to the spatial gradient is given by:

$$
\theta=\arctan (G y / G x)
$$

In this case, orientation 0 is taken to mean that the direction of maximum contrast from black to white runs from left to right on the image, and other angles are measured anti-clockwise from this [10]. 


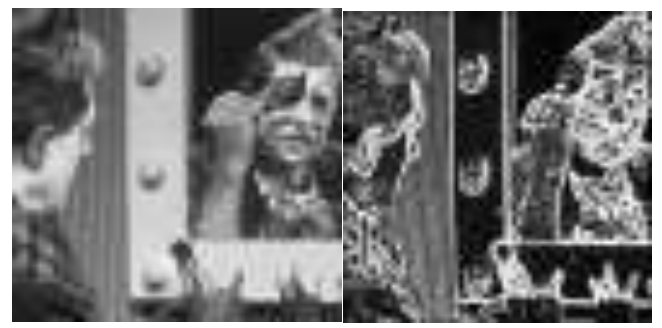

Result show the image and the results of applying the Sobel operator

\section{LAPLACIAN FILTER}

Since the input image is represented as a set of discrete pixels, we have to find a discrete convolution kernel that can approximate the second derivatives in the definition of the Laplacian. Two commonly used small kernels are shown in Figure (1.8).

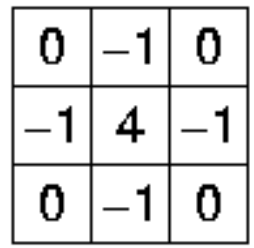

\begin{tabular}{|r|r|r|}
\hline-1 & -1 & -1 \\
\hline-1 & 8 & -1 \\
\hline-1 & -1 & -1 \\
\hline
\end{tabular}

Figure 1.8: laplacian filter

Figure (1.8) Two commonly used discrete approximations to the Laplacian filter. (Note, we have defined the Laplacian using a negative peak because this is more common; however, it is equally valid to use the opposite sign convention.) Using one of these kernels, the Laplacian can be calculated using standard convolution methods. Because these kernels are approximating a second derivative measurement on the image, they are very sensitive to noise [11].

\section{STATISTICAL MEAURES AS DISCRIMINATING FEATURES}

The statistical measures, such as Mean, Standard Deviation,Variance, Energy, Entropy is typically useful to representation characteristics or features of data, it is simple and needs minimal calculation load [12,13].

1. Mean: Is a set of intensity values for the elements of image are divided by the number, and is represented by the following equation: -

$$
\mu=\frac{1}{M N} \sum_{i=1}^{M} \sum_{j=1}^{N} x(i, j),
$$

2. Standard Deviation: The Standard Deviation is the most utilized indicator of variability and is a measurement concerning to the average distance of the scores from their mean value. It is computed on the basis of the positive square root of the variance, and is represented by the following equation: -

$$
\sigma=\sqrt{\frac{1}{M N} \sum_{i=1}^{M} \sum_{j=1}^{N}(x(i, j)-\mu)^{2}}
$$

The standard deviation is significant in detecting the details contented in an image. 
3. Variance: Is the average of the total square deviations values for the arithmetic mean. In fact it is almost identical to the standard deviation, and is represented the following equation: -

$$
\sigma^{2}=\frac{1}{M N} \sum_{i=1}^{M} \sum_{j=1}^{N}(|x(i, j)|-\mu)^{2}
$$

4. Energy: Is intended power to distribute information in the image, which is usually the factor that image is approaching compressibility, and is represented by the following equation: -

$$
E N E=\frac{1}{M N} \sum_{i=1}^{M} \sum_{j=1}^{N} x^{2}(i, j)
$$

5. Entropy: Numerical value representing the entropy of the gray image. Entropy is a statistical measure of the random that could be utilized to describe the texture information of the input image, and is represented by the following equation: -

$$
E N T=-\sum_{i=1}^{M} \sum_{j=1}^{N}\left(x(i, j) \log _{2}(x(i, j))\right.
$$

where $\mathrm{N}$ and $\mathrm{M}$ are size of row and column of the $\mathrm{x}(\mathrm{i}, \mathrm{j})$, a wavelet coefficient.

\section{THE PROPOSED SYSTEM}

The main idea for proposed system that using psoriasis images and preform preprocessing through using single DWT (Discrete Wavelet Transform) and subset of band filtered images containing wavelet coefficients and apply set of filters separately (sharpening, soble, laplacian) on low low band (LL) and using PSNR equation to show the effect of this filter on image properties. and then feature extraction using algorithm depends on wavelet transforms and apply a set of simple measures such as (Entropy, Energy, Standard derivation, Variance and Mean) to allow the user form compact and meaningful feature vectors. The proposed system passes through two main phases the (pre-processing phase and feature extraction phase). The proposed system is done in following phases:

\section{- Pre-processing Phase}

1. Input step

- Loading color image

- Convert it to gray one

- Implement single DWT (Discrete Wavelet Transform)

- Deal with three types of filters separately (sharpening, soble, laplacian)

- Make proposed using PSNR equation to show the effect of this filter on image properties.

2. Output step

- $\quad$ Compute PSNR for each case with different images

\section{- Feature extraction Phase}

- DWT (Discrete Wavelet Transform) one level and apply a set of simple measures such as (Entropy, Energy, Standard derivation, Variance and Mean).

In this paper, each phases in the proposed system will explained with the results. The figure (1.9) illustrates each phases for the proposed syste. 


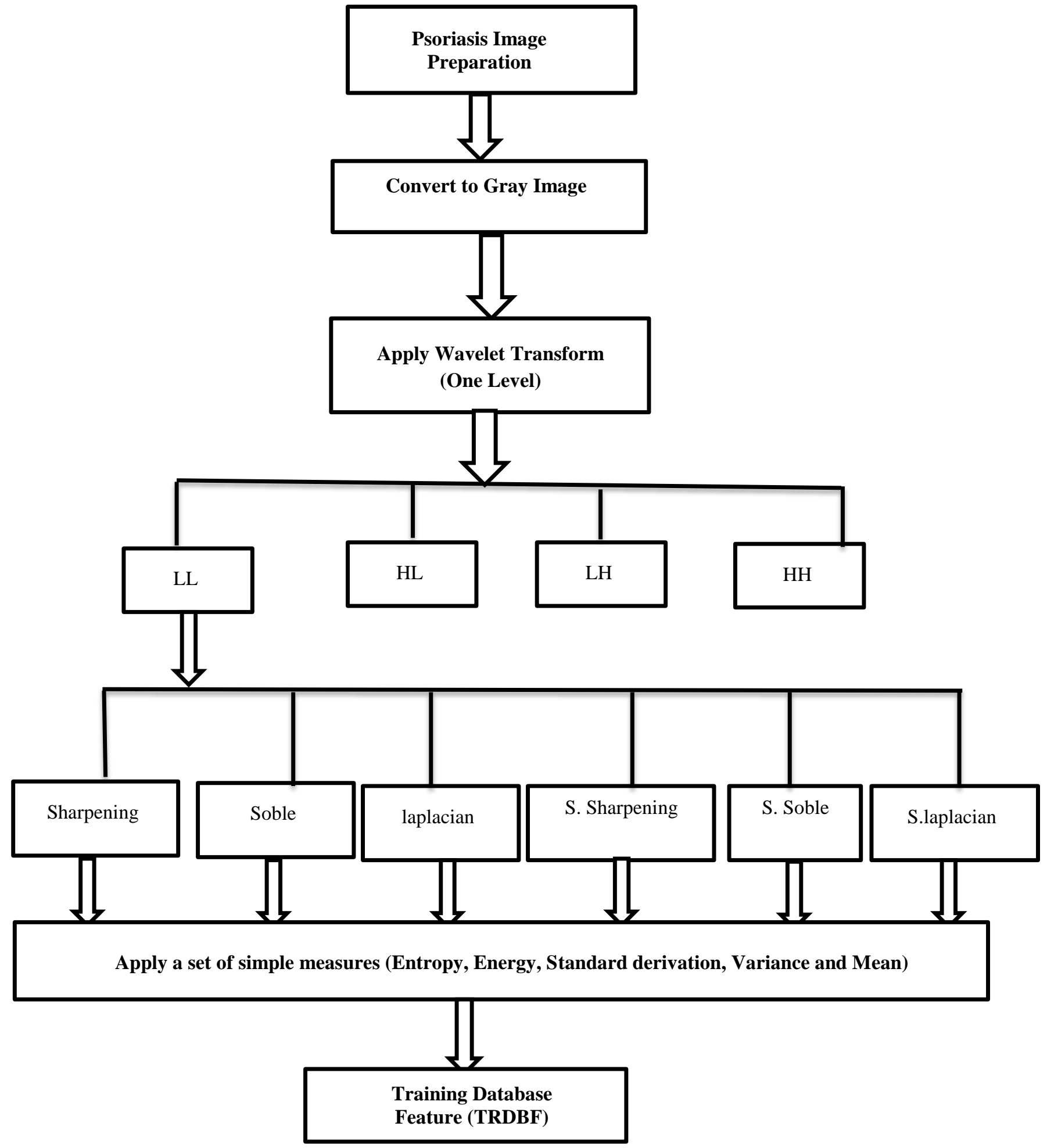

Figure 1.9: The proposed system for Psoriasis Images

\subsection{Loading Psoriasis Images}

The color psoriasis image is fed to the system as a BMP image file; the color resolution of the image is taken as 24 bit/pixel. When BMP image file is loaded, the image header information is being read as first, then, the bitmap pixel data will be read. The three bands: red, green and blue are loaded and put into 3D array with record. Figure (1.10) presents the array. The algorithm (1.1) presents the applied steps for image loading task. 


\begin{tabular}{|c|c|c|c|c|c|c|c|c|c|}
\hline \multirow{3}{*}{$\begin{array}{c}\text { No } \\
\text { Pixels }\end{array}$} & \multirow{3}{*}{$\begin{array}{c}\text { Row/ } \\
\text { Column } \\
(\mathrm{x}, \mathrm{y})\end{array}$} & \multicolumn{8}{|c|}{$\mathrm{Z}$ (Preprocessing on $\mathrm{x}$ and $\mathrm{y}$ ) } \\
\hline & & \multicolumn{3}{|c|}{ Value } & \multicolumn{3}{|c|}{ HSV } & \multirow[b]{2}{*}{ SkinHSV } & \multirow[b]{2}{*}{ Contrast } \\
\hline & & Red & Green & Blue & $\mathrm{H}$ & $\mathrm{S}$ & V & & \\
\hline \multirow{3}{*}{1} & 1,1 & & & & & & & & \\
\hline & 1,2 & & & & & & & & \\
\hline & $\cdots$ & & & & & & & & \\
\hline \multirow{3}{*}{2} & 2,1 & & & & & & & & \\
\hline & 2,2 & & & & & & & & \\
\hline & $\ldots$ & & & & & & & & \\
\hline
\end{tabular}

Figure 1.10: Example of 3D array contents

where

$\mathrm{X}$ : row pixel image, the value of $\mathrm{x}$ (0 to Image Width-1)

Y: column pixel image, the value of y (0 to Image High -1)

$\mathrm{Z}$ : containing the values of three bands also all preprocessing on $\mathrm{x}$ and $\mathrm{y}$ (the value of Red, Green, Blue, HSV, SkinHSV, ...etc).

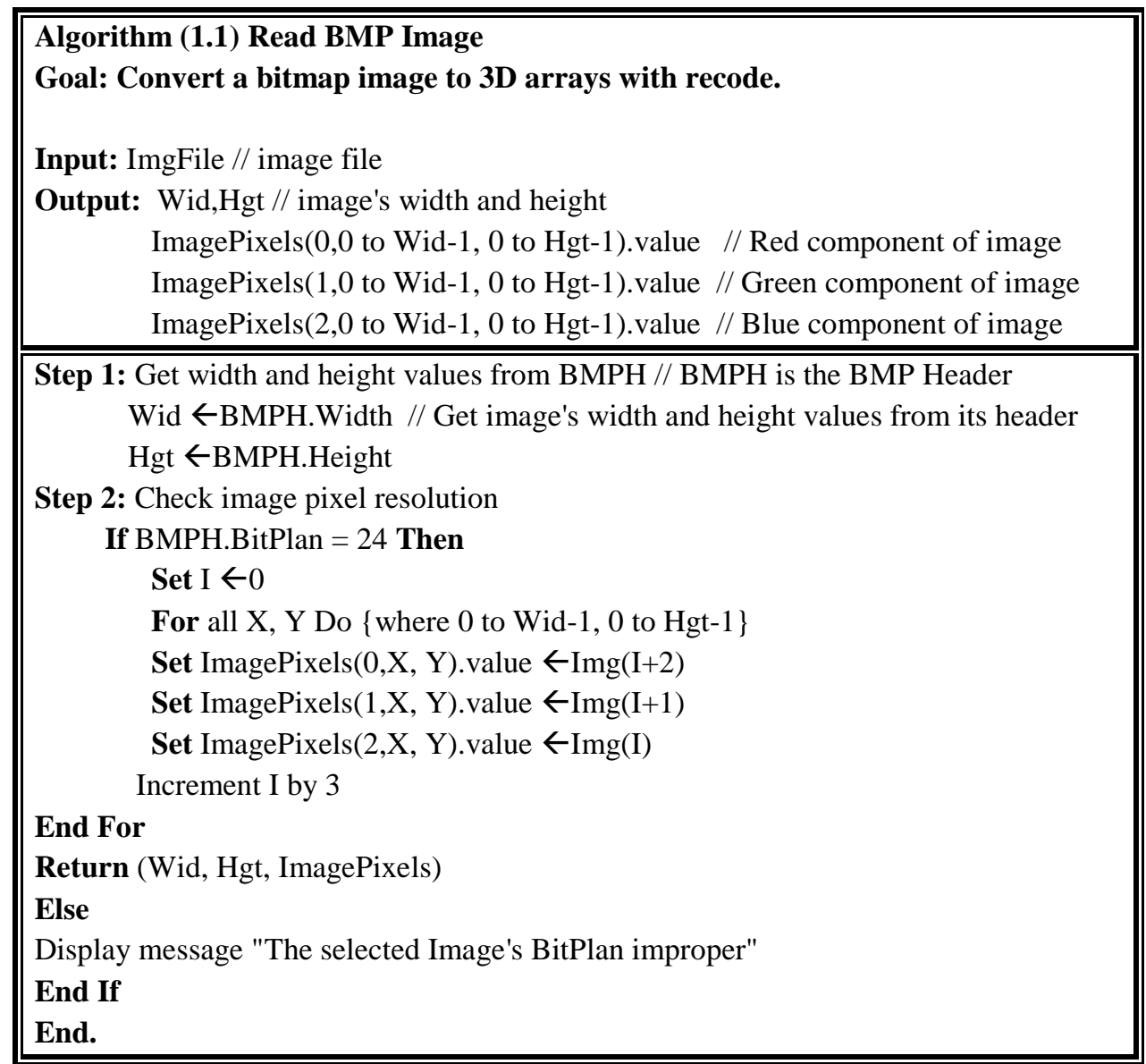




\subsection{Convert to Gray Scale}

The colored image of psoriasis will be converted to gray scale. Each colored input will be converted to gray scale image (this operation will convert 24-bit/pixel images to 256 gray scale image), the equation (15) is used in this step of proposed system. The figure below explains the result of applying threshold value in the range (127) with the sigma value equal to (0.8) of Gaussian filter to obtain best results as mentioned in algorithm (1.2).

$$
f(x, y)=\text { Thresholds }\left(\frac{R+G+B}{255}\right),
$$

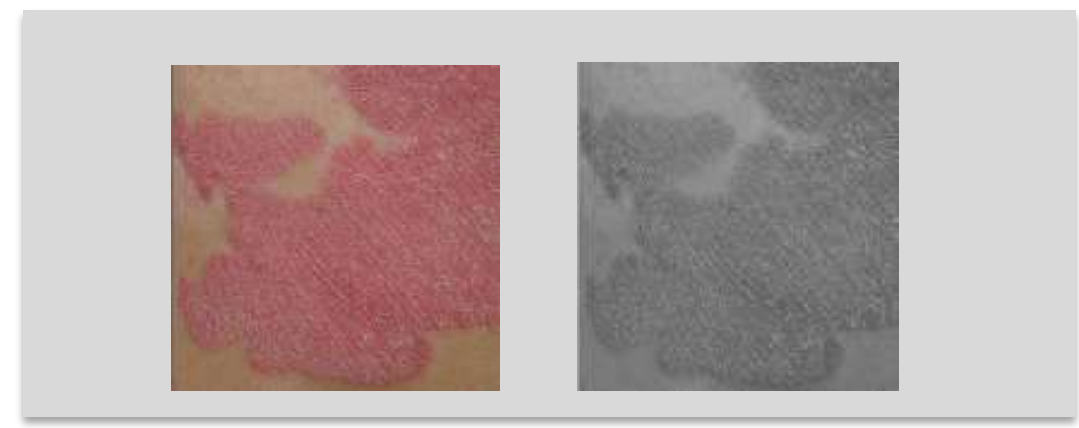

Figure 1.11: An example of result of applying thresholds:

(a) Psoriasis Image after Gaussian filter

(b)Psoriasis Image after converting to Gray-Scale

\begin{tabular}{|c|}
\hline $\begin{array}{l}\text { Algorithm (1.2) Grayscale } \\
\text { Input: Color psoriasis image (Red, Green and blue output of Gaussian iris image) } \\
\text { Output: Grayscale component of iris image }\end{array}$ \\
\hline $\begin{array}{l}\text { Step 1: Set Threshold } \leftarrow 127 \\
\text { Step 2: For all } \mathrm{X}, \mathrm{Y} \text { Do }\left\{\text { where } 0 \text { to } \mathrm{C}_{-} \mathrm{F}_{-} \text {Wid-1, } 0 \text { to } \mathrm{C}_{-} \mathrm{F}_{-} \text {Hgt-1 }\right\} \\
\text { Step 3: Set Red } \leftarrow \text { ImagePixels }(0, \mathrm{X}, \mathrm{Y}) \text {.Gaussian } \\
\text { Step 4: Set Green } \leftarrow \text { ImagePixels }(1, \mathrm{X}, \mathrm{Y}) \text {.Gaussian } \\
\text { Step 5: Set Blue } \leftarrow \text { ImagePixels }(2, \mathrm{X}, \mathrm{Y}) \text {.Gaussian } \\
\text { Step 6: Set ImagePixels }(1, \mathrm{X}, \mathrm{Y}) \text {.Grayscale } \leftarrow \text { Threshold } *\left(\frac{\text { Red }+ \text { Green }+ \text { Blue }}{255}\right) \\
\text { Step 7: End for } \\
\text { Step 8: Return (value of grayscale for this psoriasis image }) \\
\text { Step 9: End. }\end{array}$ \\
\hline
\end{tabular}

\subsection{Implement single DWT (Discrete Wavelet Transform)}

In this phases, psoriasis image will apply discrete Wavelet Transform (One Level). Figure (1.12) explains images preprocess through discrete Wavelet Transform (One Level)
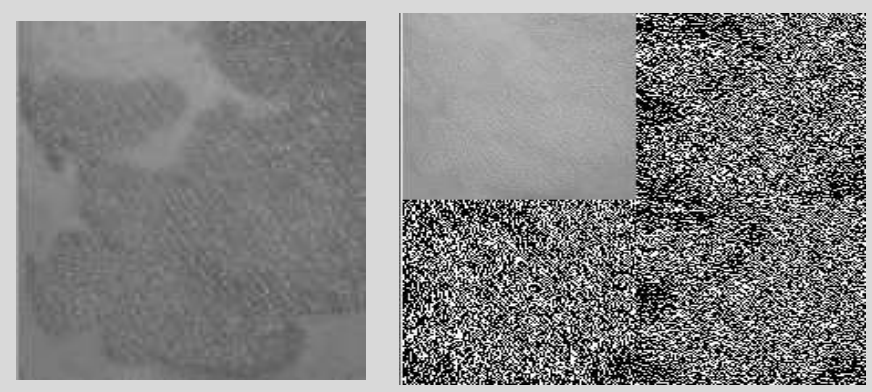

Figure 1.12: Show discrete Wavelet Transform (One Level) 
International Journal of Engineering Research And Advanced Technology, Vol.6, Issue 8, August-2020

\subsection{Apply Set of filters}

In this phase will deal with three types of filters separately (sharpening, soble, laplacian). Figure (1.13) show result apply set of filters.

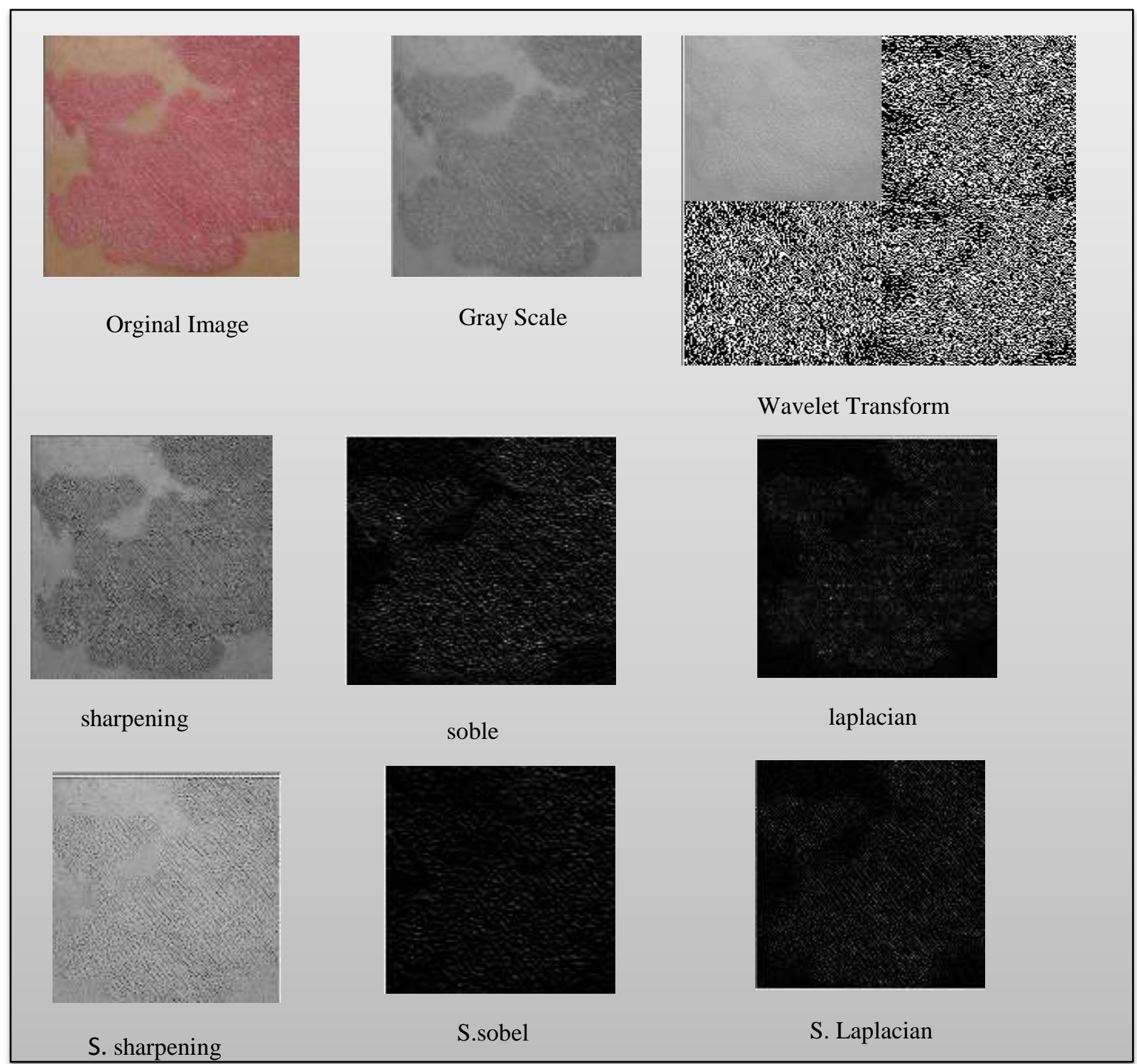

Figure 1.13: Show result apply set of filters.

\subsection{Output step}

In this phase will compute PSNR for each case with different images. Figure (1.14) show PSNR Rule and the table (1.1) show result PSNR for some sample of psoriasis image through apply set of filters of low low sub image.

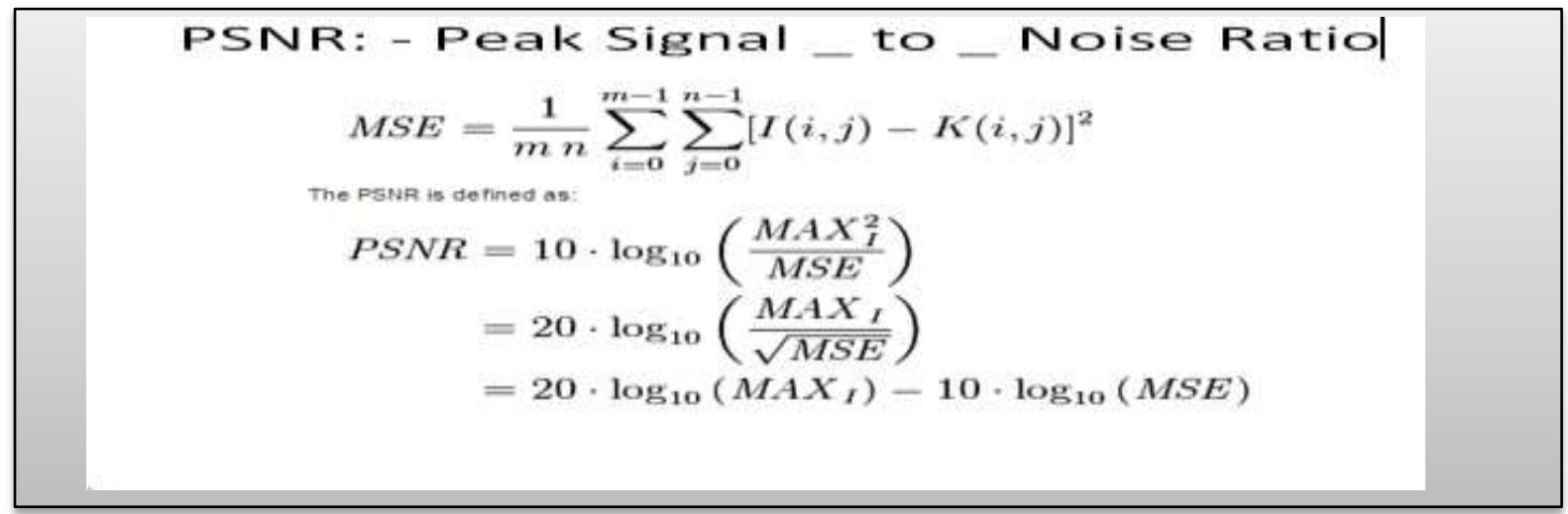

Figure 1.14: PSNR Rule 
International Journal of Engineering Research And Advanced Technology, Vol.6, Issue 8, August-2020

Table 1.1: Show result PSNR for some sample of psoriasis image

\begin{tabular}{|c|c|c|c|c|c|c|}
\hline \multirow{2}{*}{$\begin{array}{c}\text { IMAGE } \\
\text { ID }\end{array}$} & \multicolumn{5}{|c|}{ PSNR } \\
\cline { 2 - 7 } & SHARPENING & SOBEL & LAPLACE & S.SHARPENING & S.SOBEL & S.LAPLACE \\
\hline 1 & 21.31 & 0.56 & 0.25 & 11.82 & 0.58 & 0.47 \\
\hline 2 & 14.62 & 1.07 & 0.58 & 11.51 & 0.79 & 0.76 \\
\hline 3 & 26.20 & 0.46 & 0.23 & 13.33 & 0.39 & 0.33 \\
\hline 4 & 14.97 & 0.89 & 0.62 & 12.37 & 0.49 & 0.67 \\
\hline 5 & 20.22 & 0.47 & 0.28 & 12.63 & 0.44 & 0.40 \\
\hline
\end{tabular}

\section{Feature extraction Phase}

In this phase will extracted features from wavelet coefficients by applied a set of simple measures are stored in database which is called (TRDBF). This database includes (5) attributes for features such as (Energy, Entropy, Standard deviation, Variance, Mean) will apply this measures of low low sub image after apply preprocessing set of filters (sharpening, soble, laplacian) will obtain of 30 vector features for each low low sub image. Tables (1.2) and (1.3) show features for some sample of low low sub image for psoriasis image.

Table 1.2: Result of Extracted Texture Features for Some Sample of low low sub image Database (Before Normalization)

\begin{tabular}{|c|c|c|c|c|c|c|}
\hline Class & \multicolumn{6}{|c|}{ Features } \\
\hline \multirow{7}{*}{$\mathrm{C} 1$} & Sub-Image & $\begin{array}{c}\text { Energy } \\
\text { (A) }\end{array}$ & $\begin{array}{l}\text { Entropy } \\
\text { (B) }\end{array}$ & $\begin{array}{c}\text { Standard } \\
\text { Deviation (C) }\end{array}$ & $\begin{array}{l}\text { Variance } \\
\text { (D) }\end{array}$ & $\begin{array}{l}\text { Mean } \\
\text { (E) }\end{array}$ \\
\hline & Sharping (LL) & 22384.3294666667 & 6.79902761816547 & 12673.9911052357 & 1204725379016.95 & 1085085 \\
\hline & S.Sharping (LL) & 18477.5374666667 & 2.95283119743115 & 6455.92914435547 & 312592658377.037 & 552725 \\
\hline & Sobel (LL) & 26251.2262666667 & 4.49195812723266 & 9375.57767574589 & 659260925654.585 & 802691 \\
\hline & S.Sobel (LL) & 12951.3536 & 2.12910350767231 & 4503.62165637246 & 152119560178.102 & 385578 \\
\hline & Laplacian (LL) & 23253.9010666667 & 3.25525820049808 & 8126.48726739831 & 495298464803.901 & 695750 \\
\hline & S. Laplacian (LL) & 21069.7606666667 & 3.07130265667185 & 7356.93843386483 & 405934073397.581 & 629865 \\
\hline
\end{tabular}

Table 1.3: Result of Extracted Texture Features for Some Samples of low low sub image Database (After Normalization)

\begin{tabular}{|c|c|c|c|c|c|c|}
\hline Class & \multicolumn{7}{|c|}{ Features } \\
\hline \multirow{4}{*}{} & Sub-Image & $\begin{array}{c}\text { Energy } \\
(\mathbf{A})\end{array}$ & $\begin{array}{c}\text { Entropy } \\
(\mathbf{B})\end{array}$ & $\begin{array}{c}\text { Standard } \\
\text { Deviation (C) }\end{array}$ & $\begin{array}{c}\text { Variance } \\
(\mathbf{D})\end{array}$ & $\begin{array}{c}\text { Mean } \\
(\mathbf{E})\end{array}$ \\
\cline { 2 - 8 } & Sharping (LL) & 0.715 & 0.3974 & 1 & 1 & 1 \\
C1 1 & S.Sharping (LL) & 0.6712 & 0.2563 & 0.9442 & 0.9197 & 0.9533 \\
\cline { 2 - 8 } & Sobel (LL) & 0.2093 & 0.0799 & 0.5562 & 0.4519 & 0.6285 \\
\cline { 2 - 8 } & S.Sobel (LL) & 1 & 0.9604 & 0.8273 & 0.1756 & 0.3442 \\
\cline { 2 - 8 } & Laplacian (LL) & 0.9186 & 0.472 & 0.3988 & 0.062 & 0.1659 \\
\cline { 2 - 8 } & S. Laplacian (LL) & 0.6333 & 0.2655 & 0.2677 & 0.037 & 0.1114 \\
\hline
\end{tabular}




\section{THE COLLECTED DATA}

In this system proposed some of them were obtained from the hospitals and the other were obtained from the dataset (Light Field Image of Dataset Skin Lesions), were used. Figure (1.15) presents the samples of psoriasis Images. The proposed system was implemented on a medical case for psoriasis image dataset, some of them were obtained from the hospitals and the other was obtained from the dataset (Light Field Image of Dataset Skin Lesions), available in the Internet. The number of collected images are 140 they are color psoriasis Images image with $24 \mathrm{bit} / \mathrm{pixel}$ and the type of them are: BMP, JPEG, and PNG. With various image sizes.

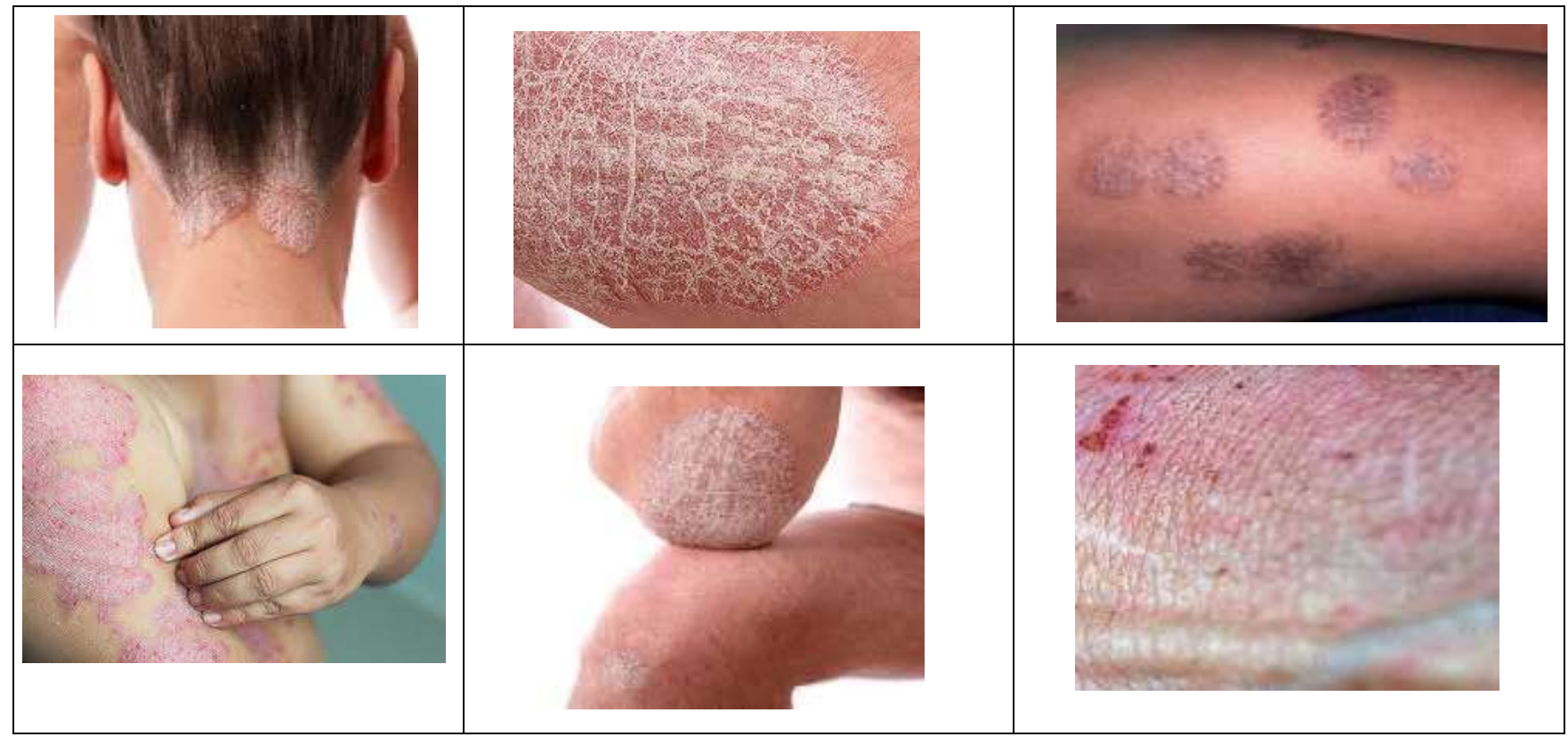

Figure 1.15: Samples of different types of psoriasis image

\section{CONCLUSIONS}

In this paper we start with simple operation so as to prepare our image which describe psoriasis case by passing it to different types of filter each one has its own property differ from the others in order to get suitable form of image at which degree of noise as least as possible then prepare it to neural or nozzle filter for identification or recognition. As we see PSNR value increase after wavelet transform for laplacian filter and this mean decrease noise. Also Using the wavelet transform to extract texture features leads to good outcomes because this technique is strong to extract texture features from psoriasis image The wavelet transform employed for feature extraction which shows robustness against secondary variances in the image sample (i.e. scaling, lighting conditions, and noise).

\section{ACKNOWLEDGMENT}

The authors would like to thank Mustansiriyah University (www.uomustansiriyah.edu.iq) Baghdad - Iraq for its support in the present work.

\section{REFERENCES}

1. R. C. Gonzalez and R. E. Woods (2002). Digital Image Processing 2/E Upper Saddle River, NJ: Prentice Hall, pp. [349- 404].

2. P. Sharma and M. Kaur (2013). Classification in Pattern Recognition: A Review. International Journal of Advanced Research in Computer Science and Software Engineering, Volume 3, Issue 4, pp. 298-306.

3. S. Bharadwaj and M.Vatsa, and R. Singh (2014). Aiding Face Recognition with Social Context Association Rule based Re-Ranking, IIIT-Delhi, India.

4. R. Kapoor, P. Mathur (2013). Face Recognition Using Moments and Wavelets", International Journal of Engineering Research and Applications (IJERA), ISSN: 2248-9622, Vol. 3, Issue 4, pp. 82-95. 
International Journal of Engineering Research And Advanced Technology, Vol.6, Issue 8, August-2020

5. Color Spaces, Apple Computer, Inc., 2006, Web Site: http://developer.apple.com/documentation/mac/ACI/ACI-3.html

6. G. R. Kumar, G. A. Ramachandra and G. Sunitha (2011). An Evolutionary Algorithm for Mining Association Rules Using Boolean Approach. IJCES International Journal of Computer Engineering Science, Volume1 Issue 3.

7. Raniah Ali Mustafa, Kawther Thabt Saleh and Haitham Salman Chyad (.2018). Feature Extraction Based on Wavelet Transform and Moment Invariants for Medical Image. International Journal of Engineering Research and Advanced Technology (IJERAT), E-ISSN : 2454-6135, Volume.4, Issue 8.

8. B.L. Zhang, H. Zhang and S. S. Ge (2004). Face Recognition by Applying Wavelet Subband Representation and Kernel Associative Memory, IEEE Transactions on Neural Networks, Vol.15, No.1.

9. B.L. Zhang, H. Zhang and S. S. Ge (2004). Face Recognition by Applying Wavelet Subband Representation and Kernel Associative Memory. IEEE Transactions on Neural Networks, Vol.15, No.1.

10. B. Jain, S. Jain and R.K. Nema, "Investigations on Power Quality Disturbances Using Discrete Wavelet Transform", International Journal of Electrical, Electronics and Computer Engineering, ISSN No. (Online): 2277-2626, 2013.

11. Fan Chung and Wenbo Zhao. A sharp Page Rank algorithm with applications to edge ranking and graph sparsification. University of California, San Diego La Jolla, CA 92093 ffan,w3zhaog@ucsd.edu.

12. A. W. S. Ibrahim (2005). Face Recognition Using Skin Color and Texture Features. Ph.D. Thesis, Department of Computer Sciences of the University of Technology.

13. Z. M. Hussain (2006). Development of a Face Recognition System Based on Canonical Correlation Analysis. Ph.D.Thesis, Informatics Institute for Postgraduate Studies at the Iraqi Commission for Computers and informatics. 